\title{
Submitted:
07.01 .2020 A case of ultrasound diagnosis of retroperitoneal lesion Accepted: caused by unnoticed foreign body ingestion
} 17.04.2020

Published: 15.06.2020

\section{Keywords}

gastric perforation, foreign body ingestion, toothpick

\author{
Wojciech Tynecki ${ }^{1}$, Andrzej Tynecki ${ }^{1}$, Agata Grobelna ${ }^{1}$, \\ Tomasz Baranowski², Grzegorz Siemiaszko', Tomasz Łapiński \\ Julia Wilczek ${ }^{1}$ \\ ${ }^{\prime}$ Gastroenterology Ward, L. Rydygier Provincial Hospital, Suwatki, Poland \\ ${ }^{2}$ E. Rittler District Hospital, Sejny, Poland \\ Correspondence: Wojciech Tynecki, Oddziat Gastroenterologii, Szpital Wojewódzki \\ im. L. Rydygiera w Suwatkach, ul. Szpitalna 60, 16-400 Suwatki, Poland, tel. +48 602288 666, \\ e-mail:drtynecki@gmail.com
}

DOI: $10.15557 /$ JoU.2020.0024

\begin{abstract}
We report the case of a 52-year-old patient who was initially admitted to the District Hospital's Surgery Ward with strong abdominal pain. The patient's medical history before the hospitalization was unremarkable. The man did not recall any worrisome situations before the onset of pain. During the hospital stay, he underwent an ultrasound scan, computed tomography and magnetic resonance imaging evaluations, and laboratory tests suggesting that paraduodenal abscess or gastrointestinal stromal tumor should be considered. The patient was referred to the provincial hospital in order to undergo endoscopic ultrasonography. This was when a retroperitoneal foreign body, a toothpick, was found and removed.
\end{abstract}

\section{Introduction}

Even though cross-sectional contrast-enhanced methods have increased in prevalence over the past 20 years, the rare case described below reaffirms that sonographic evaluation continues to play an important role in the diagnostic algorithm.

A 52-year-old male patient, with a history of obesity-related health problems, was admitted to hospital to undergo an EUS (endoscopic ultrasonography) procedure for a suspected retroperitoneal tumor.

\section{Case report}

Two weeks before the described admission, the patient was hospitalized in the Surgery Ward of a district hospital because of strong abdominal pain. On the first day of admission, laboratory tests were performed, showing a high serum CRP level (193 mg/l) and an elevated WBC count with accompanying normal serum pancreatic enzyme activity, bilirubin, AST, ALT and GGTP levels. An US (ultrasound) examination showed a hypoechoic, polycyclic lesion of unknown origin, adjacent to the pancreatic body and tail, measuring $35 \times 28 \times 50 \mathrm{~mm}$.

Subsequently, an abdominal CT (computed tomography) scan was performed, suggesting a large pathological mass, $50 \times 40 \times 50 \mathrm{~mm}$ in size, neighboring the superior wall of the D3 part of the duodenum, superior mesenteric artery, pancreatic body, with preserved pancreatic margins and with the presence of lymph nodes inside the mass and in the surrounding area. The radiologist's suggestion was either paraduodenal abscess or GIST (gastrointestinal stromal tumor). In addition, an abdominal MRI scan was performed, showing homogenous contrast enhancement of the lesion, low ADC (apparent diffusion coefficient) map signal and restricted diffusion - more consistent with GIST than abscess lesion. During this first hospitalization, the patient 


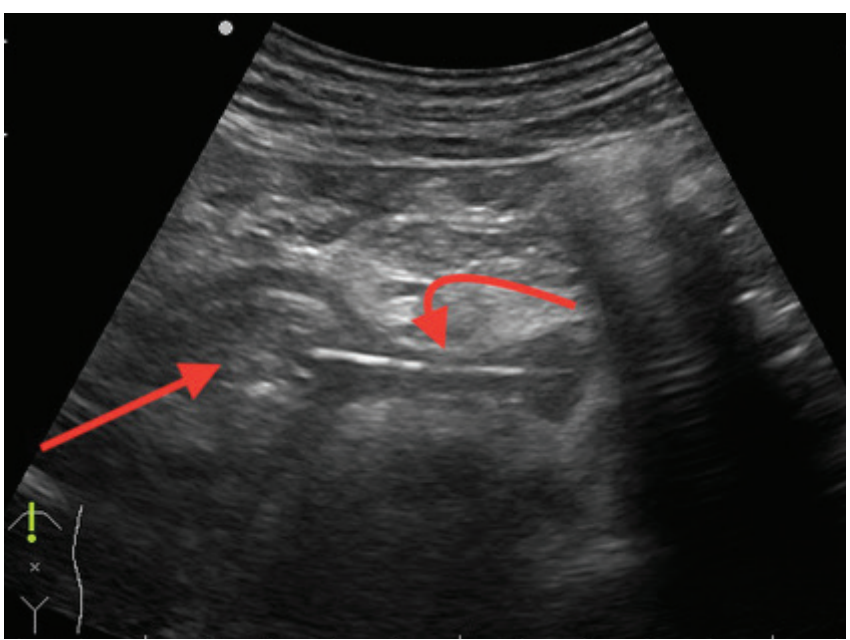

Fig. 1. Transabdominal ultrasound, Aloka Alpha Prosound 6, straight arrow: stomach (pyloric part) transverse view, curved arrow: toothpick

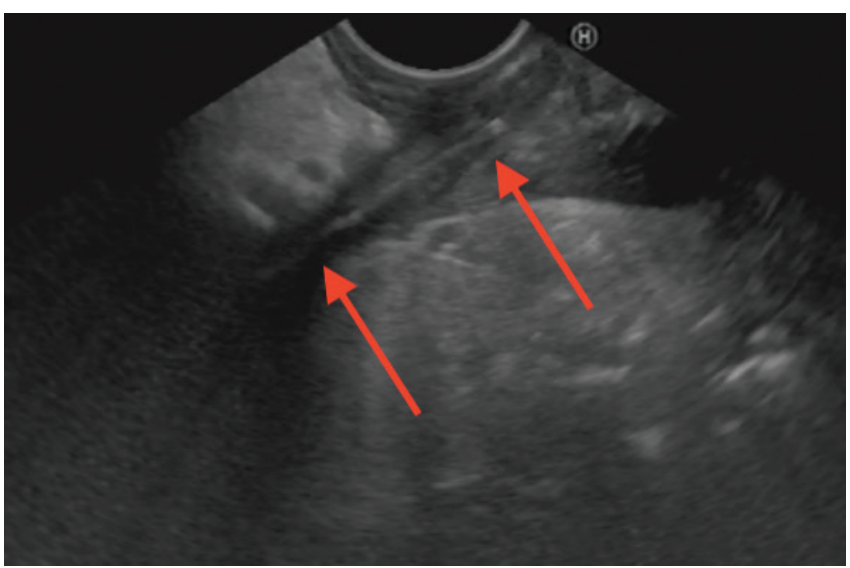

Fig. 2. Endoscopic ultrasound, Hitachi Avius, Pentax EG-3870 UTK, transgastric approach, arrows: toothpick

was treated with antibiotics with good effect. The course of medication resulted in a decrease in serum CRP levels and a reduced sensation of pain.

Because of improvement in his condition and for personal reasons, the patient delayed the EUS-related hospitalization for nearly two weeks, showing up asymptomatic and with normal laboratory results on the day of admission. It was then that a transabdominal US scan was performed, showing a linear hyperechoic object (40 $\mathrm{mm}$ in length) extending from the gastric antrum wall (muscular layer), and heading through the retroperitoneal site in the direction of the pancreatic body, surrounded by a dense fluid collection. The findings were consistent with the presence of a foreign body (Fig. 1). The patient was once again asked about his medical history and recent symptoms. Initially, he could not recall any probable situation of ingesting a foreign body.

In the next step, gastroscopy was performed endoscopically, showing no obvious abnormalities. An EUS ultrasound image confirmed the presence of a linear foreign body stuck (5.6 mm deep from the ultrasound probe surface) in the muscular layer of the antrum. A precise evaluation of the extent of the lesion and surrounding structures was described (Fig. 2).

Gastroscopy was performed to determine the probable penetration area, and slightly lifted mucosa was noticed within the suspected area. After pushing it with biopsy forceps, a small amount of pus was released, and the mucosa collapsed, eliminating the possibility of assessing the site endoscopically.

A surgical consultation was held, and the patient was considered eligible for laparotomy. During the procedure, a wooden toothpick measuring $40 \mathrm{~mm}$ was extracted (Fig. 3).

\section{Conclusions}

This is a rare case of unnoticed ingestion of a foreign body resulting in alimentary tract perforation with total displacement of the object out of the tract lumen. The case shows that sonographic evaluation still plays an important role in the diagnostic algorithm, even after cross-sectional contrast-enhanced modalities have been employed. Moreover, in the reported case, repeating the transabdominal sonographic evaluation and supplementing it with endosonography, contributed to making the correct diagnosis. With its ease of use and safety, US can be successfully used to verify the diagnosis at every stage of the therapeutic and diagnostic processes. Its main disadvantage continues to be operator dependency.

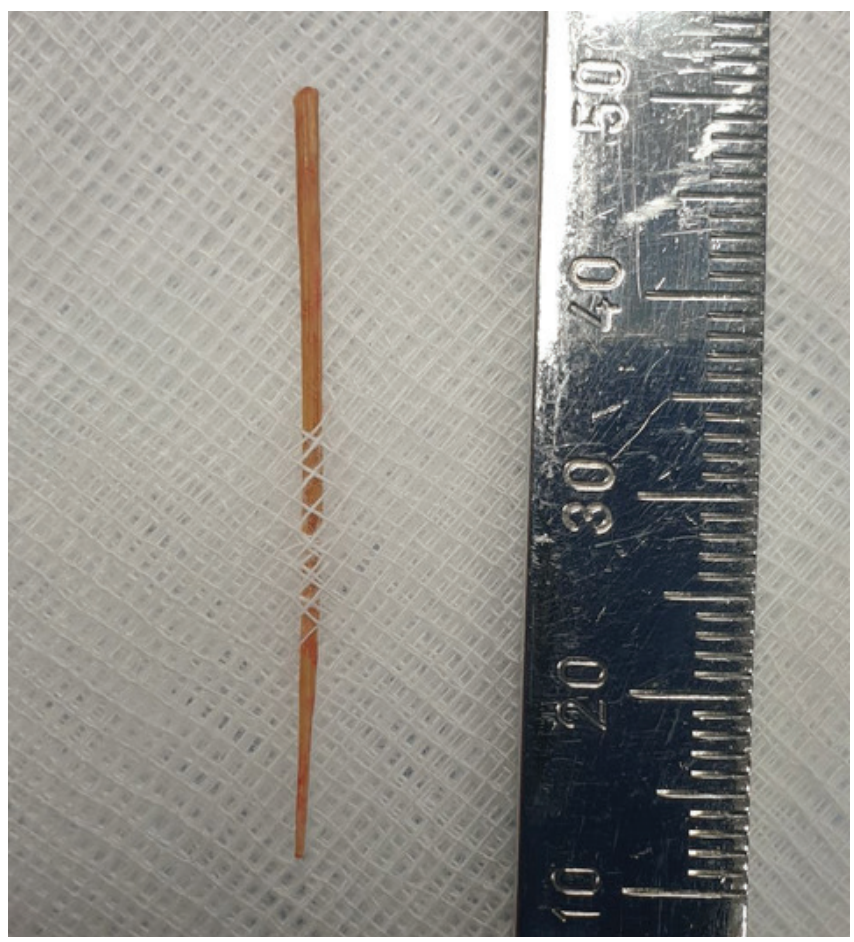

Fig. 3. $40 \mathrm{~mm}$ toothpick removed by the surgery team 


\section{Discussion}

Ingestion of foreign bodies has the greatest prevalence in children (about $80 \%$ of cases) ${ }^{(1)}$, and nearly $80 \%$ of all ingested objects pass spontaneously through the tract in 4-6 days without any medical assistance, about $20 \%$ of cases need an endoscopic procedure, and less than $1 \%$ of cases need surgical treatment ${ }^{(2)}$. In children, the most common foreign bodies found in the alimentary tract include coins, buttons, button batteries and toys, while in adults the impaction of an object is usually associated with food (food boluses, fish bones, chicken bones), though foreign bodies like dentures are also occasionally observed. There are only a few sites one can expect an ingested object to impact due to the natural narrowing of the lumen and angulations, including the esophageal sphincters, pylorus, duodenal sweep, ileocecal valve, and anus ${ }^{(1-3)}$. When the nature, size and number of obstacles are unknown, or a radiopaque object is suspected, a plain radiograph is recommended in the first place ${ }^{(4)}$. In cases involving sharp objects,

\section{References}

1. Bekkerman M, Sachdev AH, Andrade J, Twersky Y, Iqbal S: Endoscopic management of foreign bodies in the gastrointestinal tract: a review of the literature. Gastroenterol Res Pract 2016; 2016: 8520767.

2. Birk M, Bauerfeind P, Deprez PH, Häfner M, Hartmann D, Hassan C et al.: Removal of foreign bodies in the upper gastrointestinal tract in adults: European Society of Gastrointestinal Endoscopy (ESGE) Clinical Guideline. Endoscopy 2015; 47: 1-46. stretching tract walls and continuous peristaltic movements may lead to perforation and penetration into surrounding tissues. If such a suspicion arises, a CT scan should be consid$\operatorname{ered}^{(2)}$. MRI and ultrasound examinations are not routinely used in the diagnostic algorithm for foreign body ingestion, but in the reported case ultrasound and endoscopic ultrasound assessments played a major role in the differential diagnostic process.

Some sources claim that sonography seems to be the most helpful method for evaluating retained wooden objects ${ }^{(5)}$.

\section{Conflict of interest}

The authors do not report any financial or personal connections with other persons or organizations which might negatively affect the contents of this publication and/or claim authorship rights to this publication.
3. Tammana VS, Valluru N, Sanderson A: All the wrong places: an unusual case of foreign body ingestion and inhalation. Case Rep Gastroenterol 2012; 6: 778-783.

4. ASGE Standards of Practise Committee, Ikenberry SO, Jue TL, Anderson MA, Appalaneni V, Banerjee S, et al.: Management of ingested foreign bodies and food impactions. Gastrointest Endosc 2011; 73:1085-1091.

5. Peterson JJ, Bancroft LW, Kransdorf MJ: Wooden foreign bodies: imaging appearance. AJR Am J Roentgenol 2002; 178: 557-562. 